\title{
Numerical stability analysis of linear stochastic delay differential equations using Chebyshev spectral continuous time approximation
}

\author{
Shahab Torkamani · Ehsan Samiei • \\ Oleg Bobrenkov • Eric A. Butcher
}

Received: 16 September 2013 / Revised: 25 February 2014 / Accepted: 1 March 2014 / Published online: 26 March 2014

(c) Springer-Verlag Berlin Heidelberg 2014

\begin{abstract}
In this paper, a numerical approach is developed for the stability analysis of linear stochastic delay differential equations (SDDEs) in the parameter space based on the Chebyshev Spectral Continuous Time Approximation (CSCTA) technique. The CSCTA method is used to approximate an infinite-dimensional linear SDDE as a set of linear stochastic differential equations (SDEs). The mean and mean-square stability concepts are employed for the stochastic stability analysis of the resulting SDE. For this purpose, a set of linear deterministic differential equations for both the first and second moments are obtained using the Ito differential rule. Two examples are provided: a first order SDDE with multiplicative stochastic excitation and a second order SDDE with both additive and multiplicative stochastic excitation. In both examples the stability charts obtained from the proposed approach match those obtained using the stochastic semi-discretization method as described by Elbeyli et al. (Commun Nonlinear Sci Numer Simul 10(1):85-94, 2005).
\end{abstract}

\section{S. Torkamani}

Department of Aerospace Engineering and Mechanics,

The University of Alabama, Tuscaloosa, AL 35408, USA

e-mail: storkamani@bama.ua.edu

\section{E. Samiei $(\varangle)$}

Department of Mechanical and Aerospace Engineering, New Mexico State University, Las Cruces, NM 88003, USA

e-mail: esamiei@nmsu.edu

O. Bobrenkov

Forest LLC, Moscow 105264, Russia

e-mail: oleg.bobrenkov@gmail.com

\section{E. A. Butcher}

Department of Aerospace and Mechanical Engineering,

University of Arizona, Tucson, AZ 85721, USA

e-mail: ebutcher@email.arizona.edu
In the first example the stability results obtained from both numerical approaches are found to be less conservative than the Lyapunov-based stability region obtained by Samiei et al. (Int J Dyn Control 1(1):64-80, 2013).

Keywords Time-delay - White noise - Brownian motion process - Chebyshev spectral continuous time approximation $\cdot$ Semidiscretization $\cdot$ Moment stability

\section{Introduction}

Stochastic phenomena can arise in various physical and engineering processes [3] such as cosmic physics [4], communication systems [5], traffic control [6], etc. They can change the behavior of systems and lead them to instability. Thus, investigating the stability of stochastic differential equations (SDEs) and modeling such systems are of a great importance. During recent decades, techniques for stability analysis of SDEs have been developed. Some background regarding the theory of stochastic systems can be found in [7-9].

Stochastic time-delayed dynamical systems have attracted increasing attention due to the instability and poor performance that a time delay can cause, and also due to possible stochastic resonance between the Kramers rate of escape from a potential well and the delay [10]. There have been a number of studies focusing on stability analysis of SDDEs in the literature [11-18]. Stochastic stability of the equilibrium solution of SDDEs can be studied from different notions of stability including asymptotic stability [11,12], exponential stability [13,19], moment stability $[14,15]$, and Lyapunov stability $[2,16,18]$. Two methods that are used to prove stability in the framework of Lyapunov's direct method include the Lyapunov-Krasovskii functional and the Lyapunov-Razumikhin function. Examples of sta- 
bility analysis of SDDEs using the Lyapunov-Krasovskii functional can be found in $[2,16]$, while examples of the Lyapunov-Razumikhin function are found in $[2,18]$. A class of numerical techniques often used to study the stability of time-delayed systems are discretization-based methods in which an extended state vector is used to approximate the infinite-dimensional state. It is known that discretization techniques preserve asymptotic stability for delay differential equations (DDEs) [20,21]. A well-known technique of this type is the semi-discretization method which was originally used in solid and fluid mechanics and adopted for stability analysis of deterministic time-delayed systems in [22]. The semi-discretization method is adapted for the stability analysis of linear SDDEs in [1], where stability boundaries and steady state second order moments were studied using this approach. Rather than using equally-spaced grid points (which is always assumed in semi-discretization), however, the essence of pseudospectral differentiation methods lies in the utilization of unevenly spaced discretization points with more points lumped near the boundaries. As shown and discussed in [23], pseudospectral differentiation has a major advantage over approximation techniques that use equally-spaced grid points in its spectrally accurate exponential convergence characteristics. The CSCTA method has been used previously in multiple studies for stability analysis $[24,25]$ as well as system identification in deterministic time delay systems [26-28]. Moreover, in $[29,30]$, the analytical convergence characteristics of the pseudospectral Chebyshev method has been performed to investigate the stability of equilibria and periodic solutions of DDEs.

In this paper, we utilize the CSCTA numerical technique to approximate a general class of linear SDDEs as a set of linear SDEs. The mean and mean-square stability concepts are then employed for the stability analysis of the resulting linear SDEs, where a set of linear deterministic differential equations for each moment is obtained using the Ito differential rule. Finally, stability boundaries corresponding to the first and second moments are calculated in the parameter space by using the eigenvalue criteria. The paper is organized as follows: in Sect. 2, linear SDDEs and the concept of moment stability is introduced. In Sect. 3, it is shown how a linear infinite-dimensional SDDE can be approximated as a set of linear finite-dimensional SDEs using the CSCTA technique. The numerical stability analysis of SDDEs using CSCTA along with the theory of moment stability is discussed in Sect. 4. Finally in Sect. 5, the proposed numerical approach is applied on first and second order SDDE systems. The resulting stability boundaries in the parameter space are compared with those obtained using a Lyapunov-based approach shown in [2] as well as using the stochastic semi-discretization method as presented in [1].

\section{Linear SDDEs and $m$ th moment stability}

In this section, we introduce linear SDDEs and review the concept of $m$ th moment stability for SDDEs. Consider a physical process modeled as a linear, time-invariant, timedelayed, system which is excited by a stochastic disturbance modeled as a continuous Gaussian wide-band process which appears in both additive and multiplicative form. The mathematical model can thus be described by

$\dot{x}(t)=A x(t)+A_{1} x(t-\tau)+\left(G_{0}+G_{1} x(t)\right) \zeta(t), t \geq t_{0}$,

$x(\theta)=\phi(\theta), t_{0}-\tau \leq \theta \leq t_{0}$,

where $x(t) \in \mathcal{R}^{n}, \zeta(t) \in \mathcal{R}^{q}$ is the stochastic disturbance, $\tau$ is the known discrete time delay, $x_{t}(\theta)=x(t+\theta) \in \mathcal{C}\left(\left[t_{0}-\right.\right.$ $\left.\tau, t_{0}\right], \mathcal{R}^{n}$ ) for $t_{0}-\tau \leq \theta \leq t_{0}$ is the infinite-dimensional state residing in a Banach space of continuous functions on the interval of length $\tau, \phi(\theta)$ is the history function on the interval $\left[t_{0}-\tau, t_{0}\right], A \in \mathcal{R}^{n \times n}$ and $A_{1} \in \mathcal{R}^{n \times n}$ are constant coefficient matrices, $G_{0} \in \mathcal{R}^{n \times q}$ is the additive random excitation coefficient, and $G_{1} \in \mathcal{R}^{n \times n \times q}$ is a third-order tensor which represents the multiplicative random excitation coefficient, i.e., $\left(G_{1} x(t) \zeta(t)\right)_{i}=\sum_{l=1}^{l=n} \sum_{k=1}^{k=q} G_{1_{i l k}} x_{l}(t) \zeta_{k}(t)$, where $i=1,2, \ldots, n$.

$\zeta(t)$ can be approximated by a zero-mean Gaussian white noise process which is defined as a vector of stochastic processes independent of the state with covariance matrix $Q(t)$, i.e. $w(t) \sim N(0, Q(t))$. A white noise stochastic process is formally defined as the derivative of a Brownian motion (Weiner) process textcolorred $\beta(t)$, i.e.,

$w(t)=\frac{d \beta(t)}{d t}$,

where $d \beta(t) \in \mathcal{R}^{q}$ is a stochastic Brownian motion increment process with $E\{d \beta(t)\}=0, E\left\{d \beta(t) d \beta^{T}(t)\right\}=$ $Q(t) d t$, and $E$ \{.\} represents the expectation operator. Note that $\beta(t)$ defined on the complete probability space $(\Omega, \mathrm{F}, \mathbb{P})$, where $\Omega$ is the sample space, $\mathrm{F}$ is the $\sigma$-algebra of subsets of the sample space, and $\mathbb{P}$ is the probability measure on $\mathrm{F}$.

Using the definition of Brownian motion, the physical form in Eq. (1) with $\zeta(t)$ approximated with $w(t)$ can be rewritten in the equivalent integral form as

$$
\begin{aligned}
x(t)= & x\left(t_{0}\right)+\int_{t_{0}}^{t}\left[A x(s)+A_{1} x(s-\tau)\right] d s \\
& +\int_{t_{0}}^{t}\left[G_{0}+G_{1} x(s)\right] d \beta(s) .
\end{aligned}
$$

Unlike the first integral which is a Riemann integral, the second integral contains a stochastic integral which may be interpreted in either the Ito or Stratonovich sense. However, because Eq. (1) represents a physical form, the Stratonovich 
interpretation is required (see $[8,9]$ for further discussion on the Ito and Stratonovich interpretations of a stochastic integral).

In order to discuss stability, however, we first transform Eq. (3) to the equivalent Ito differential form

$$
\begin{aligned}
d x(t)= & {\left[A_{0} x(t)+A_{1} x(t-\tau)+C\right] d t } \\
& +\left[G_{0}+G_{1} x(t)\right] d \beta(t), t \geq t_{0}, \\
x(\theta)= & \phi(\theta), t_{0}-\tau \leq \theta \leq t_{0},
\end{aligned}
$$

where $\left(A_{0} x(t)\right)_{i}+C_{i}=(A x(t))_{i}+\frac{1}{2} \sum_{l=1}^{l=n} \sum_{r=1}^{r=q} \sum_{k=1}^{k=q}$ $\left(G_{l r}\right) Q_{r k} \frac{\partial G_{i k}}{\partial x_{l}}$, and $G(x(t))=G_{0}+G_{1} x(t)$. The term $\frac{1}{2} \sum_{l=1}^{l=n} \sum_{r=1}^{r=q} \sum_{k=1}^{k=q}\left(G_{l r}\right) Q_{r k} \frac{\partial G_{i k}}{\partial x_{l}}$ is the Wong-Zakai correction term which appears in the Ito representation of SDEs. $t_{0}$ is assumed to be zero in the above equation and hereafter. It is worthy to note that the above transformation from Stratonovich to Ito can be found in $[8,9]$ and is formally proved in [31].

Note that $x(t)=0$ is not an equilibrium solution for Eq. (4) since there are both constant matrix $C$ and the additive noise term $G_{0} d \beta(t)$ in Eq. (4). However, we instead investigate the stability of a nominal solution $\bar{x}(t)$ for Eq. (4) satisfying

$$
\begin{gathered}
d \bar{x}(t)=\left[A_{0} \bar{x}(t)+A_{1} \bar{x}(t-\tau)+C\right] d t \\
+\left[G_{0}+G_{1} \bar{x}(t)\right] d \beta(t), t \geq 0, \\
\bar{x}(\theta)=\phi(\theta), \quad-\tau \leq \theta \leq 0 .
\end{gathered}
$$

We are interested in the stability analysis of this nominal solution. Note that $\bar{x}(t)=0$ can be considered as an equilibrium point for Eq. (4) if $C=0$ and $G_{0}=0$ (no additive noise). Let $x(t)$ be any perturbed solution of Eq. (4). To remove the constants $C$ and $G_{0}$, we define the perturbation $y(t)$ as $y(t)=x(t)-\bar{x}(t)$. Thus differentiating the perturbation using Eqs. (4) and (5) yields

$d y(t)=\left[A_{0} y(t)+A_{1} y(t-\tau)\right] d t+G_{1} y(t) d \beta(t), t \geq 0$,

for which the trivial solution $y(t)=0$ is an equilibrium point. Now, we analyze the stability of the trivial solution of Eq. (6) which implies the stability of $\bar{x}(t)$. In addition, for any $\phi(\theta) \in \mathcal{C}\left([-\tau, 0], \mathcal{R}^{n}\right),-\tau \leq \theta \leq 0$, the initial value problem of Eq. (6) (with the transformation above to $y(t)$ has the solution $y(t ; \phi)$ when it satisfies, with probability 1 , the following integral equation:

$$
\begin{aligned}
y(t)= & y(0)+\int_{0}^{t}\left[A_{0} y(s)+A_{1} y(s-\tau)\right] d s \\
& +\int_{0}^{t}\left[G_{1} y(s)\right] d \beta(s) \quad t \geq 0,
\end{aligned}
$$

where in this equation the stochastic integral is understood in the Ito sense. This solution is unique over the interval of $[0, t]$, i.e., the finite escape time is zero, since the constant coefficient matrices of $A_{0}, A_{1}, G_{1}$ are assumed to be norm bounded. Note that since Eq. (4) is a linear, time-invariant SDDE over $[0, t]$ and the coefficient matrices are bounded the uniqueness of the solution of Eq. (4) is trivial. Existence and uniqueness of solution of SDDEs appear in $[15,32,33]$.

Several different modes of stochastic stability can be defined. Lyapunov stability, stability in probability, almost sure stability, and stability in the $m$ th moment are various definitions of stochastic stability. In this paper, we are interested in $m$ th moment stability analysis of linear SDDEs, and specifically the first and second moments stability analysis. The second moment stability analysis implies stability in probability and for linear stochastic systems, as is the case for this paper, it implies almost sure stability. More details about the modes of the stochastic stability and their relations can be found in $[8,34]$.

The following Definition describes the $m$ th moment stability concept for Eq. (6) $[8,35]$.

Definition 1 The trivial solution $y=0$ of Eq. (6) is said to be stable in the $m$ th moment if for every $t_{0} \geq 0$ and $\epsilon>0$, there exists a $\delta\left(t_{0}, \epsilon\right)>0$ for Eq. (6) such that

$$
\begin{aligned}
& \sup _{-\tau \leq \theta \leq 0}\|E\{\phi(\theta)\}\|<\delta \rightarrow\left\|E\left\{y(t ; \phi)^{m}\right\}\right\|<\epsilon, \\
& \quad \forall t \geq 0, m>0,
\end{aligned}
$$

where $y(t ; \phi)^{m}=y_{1}^{s_{1}} y_{2}^{s_{2}} \ldots y_{n}^{s_{n}}, s=\left[s_{1}, s_{2}, \ldots, s_{n}\right]^{T}, \quad \sum_{i=1}^{i=n}$ $s_{i}=m$, and $\|()\|=.\left(\sum_{s}(.)^{2}\right)^{\frac{1}{2}}$. The nominal solution of Eq. (5) is said to be asymptotically stable in the $m$ th moment if Eq. (8) holds and

$\lim _{t \rightarrow \infty}\left\|E\left\{y(t ; \phi)^{m}\right\}\right\|=0$.

These two concepts can be changed to the uniform stability and uniform asymptotic stability in the $m$ th moment if the function $\delta$ is independent of $t_{0}$, i.e., $\delta(\epsilon)$. It is worth mentioning that the above definition of the moment stability represents the joint moment of order $m$ for the elements of vector $y$, i.e, joint moment of order $m$ for $n$ random variables $y_{1}, y_{2}, \ldots, y_{n}$. Moreover, after the change of variable, Definition 1 still holds and the stability of the trivial solution is not affected. The stability of the first and second moments are known as mean and mean square stability.

\section{Chebyshev spectral continuous time approximation}

In the context of functional analysis, the infinite-dimensional linear Ito SDDE of Eq. (6) can be lifted to an abstract stochastic Cauchy problem in terms of the evolution of an initial 
function $\phi=y(0)$ in a Banach space of continuous functions $\mathcal{C}\left([-\tau, 0], \mathcal{R}^{n}\right)$, i.e.

$d y(t)=(\tilde{\mathbb{A}} y(t)) d t+(\tilde{\mathbb{G}} y(t)) d \beta(t)$,

where

$$
\begin{aligned}
& \tilde{\mathbb{A}} \phi=\frac{d \phi}{d \theta}, \quad-\tau \leq \theta<0, \\
& (\tilde{\mathbb{A}} \phi) d t+(\tilde{\mathbb{G}} \phi) d \beta(0)=\left(A_{0} y(0)+A_{1} y(-\tau)\right) d t \\
& \quad+\left(G_{1} y(0)\right) d \beta(0), \quad \theta=0,
\end{aligned}
$$

in which $y(t)$ is the infinite-dimensional vector, $\tilde{\mathbb{A}}: \mathcal{C} \rightarrow \mathcal{C}$ is the generator of the $\mathcal{C}_{0}$-semigroup in the absence of stochastic excitation and $\tilde{\mathbb{G}}$ is an infinite-dimensional stochastic excitation coefficient operator. The main idea behind the continuous time approximation (CTA) technique is that the infinite-dimensional vector $y(t)$ and operators $\tilde{\mathbb{A}}$ and $\tilde{\mathbb{G}}$ can be approximated by finite-dimensional vectors and matrices. Further information on the relation between the domain and the spectrum of the solution operator can be found in [3638]. More discussions on semigroup approach to DDEs and SDDEs in Banach space is also presented in [39-42]. This represents a straight-forward extension of the abstract form of a deterministic DDE, e.g. see $[24,31,38]$. As shown and discussed in [23], spectral differentiation has a major advantage over finite difference differentiation in its spectrally accurate exponential convergence characteristics.

A finite-dimensional approximation to $y(t)$ is now defined as

$$
\begin{aligned}
Y(t) & \cong\left[Y_{1}^{T}(t), Y_{2}^{T}(t), \ldots, Y_{(N+1)}^{T}(t)\right]^{T} \\
Y_{i}(t) & \cong\left[\mathbb{Y}_{1}^{T}(t), \mathbb{Y}_{2}^{T}(t), \ldots, \mathbb{Y}_{n}^{T}(t)\right]^{T} \\
& \cong\left[y_{1}^{T}\left(t-\tau_{i-1}\right), y_{2}^{T}\left(t-\tau_{i-1}\right), \ldots, y_{n}^{T}\left(t-\tau_{i-1}\right)\right]^{T}
\end{aligned}
$$

where $\tau_{i-1}=\tau / 2\left(1-t_{i-1}\right)$ and $t_{i-1}=\cos \left(\frac{(i-1) \pi}{N}\right)$ are the unevenly spaced points corresponding to the extremum points of the Chebyshev polynomial of the first kind [43] of degree $N$ defined in the interval $[-1,1]$. Thus with the Chebyshev points being defined so, the number of collocation points will be $M=N+1$.

We now define a $M \times M$ Chebyshev spectral differentiation matrix $D$ associated with the Chebyshev points. Assuming the rows and columns of $D$ matrix are indexed from 0 to $N$, the entries of the matrix will be

$D_{00}=\left(2 N^{2}+1\right) / 6, \quad D_{N N}=-\left(2 N^{2}+1\right) / 6$,

$D_{j j}=\left(-t_{j}\right) / 2\left(1-t_{j}^{2}\right), j=1, \ldots, N-1$

$D_{i j}=c_{i}(-1)^{i+j} / c_{j}\left(t_{i}-t_{j}\right), i \neq j, i, j=0, \ldots, N$,

where $c_{i}=2$ for $i=0, N$, otherwise $c_{i}=1$. The $M n \times$ $M n$ differential operator $\mathbb{D}$ (corresponding to $n$ first order
SDDEs) is defined as $\mathbb{D}=D \otimes I_{n}$ in which $I_{n}$ is a $n \times n$ identity matrix and $\otimes$ denotes Kronecker product. Eq. (10) can be discretized and approximated by initially replacing the first $n$ rows of $\mathbb{D}$ by $A_{0}$ and $A_{1}$ to form a finite matrix $\mathbb{A}$ which approximates the operator $\tilde{\mathbb{A}}$. Also, $\mathbb{G}$ is formed by inserting $G_{1}$ in the top $n$ rows of the coefficient matrix of $d \beta(t)$ with the remaining rows equal to zero to approximate the coefficient matrix $\tilde{\mathbb{G}}$ of $d \beta(t)$, i.e.,

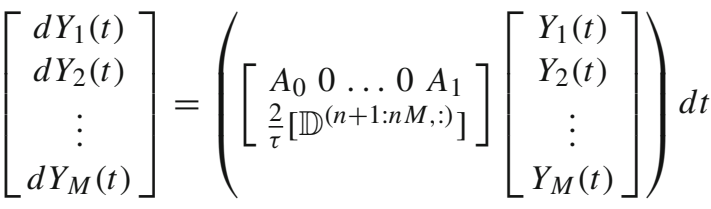

$$
\begin{aligned}
& +\left[\begin{array}{c}
G_{1} Y_{1}(t) \\
0_{n \times q} \\
\vdots \\
0_{n \times q}
\end{array}\right] d \beta(t)
\end{aligned}
$$

Note that the superscript $(n+1: M n,:)$ on $\mathbb{D}$ refers to the fact that only rows of $\mathbb{D}$ lying between $n+1$ and $M n$ are written into the remaining $n(M-1) \times M n$ elements of the matrix $\tilde{\mathbb{A}}$. The $\frac{2}{\tau}$ factor in front of $\mathbb{D}$ in Eq. (14) is a normalization factor which accounts for the rescaling of the standard collocation expansion interval $[-1,1]$ to the interval $[0, \tau]$. Therefore, the infinite-dimensional linear SDDE of Eq. (4) can be converted into a large system of finite-dimensional linear SDEs using the CSCTA technique, in which the dimension of Eq. (14) depends on the order of the Chebyshev grid used. As the estimate of the error bounds for the collocation polynomial and characteristic roots of constant-coefficient DDEs using Chebyshev collocation points studied in [29] show that the accuracy exponentially increases with the degree $N$ of the collocation polynomial. Thus, a larger grid results in better accuracy of the SDE approximation of the original SDDE.

\section{Stability analysis of CSCTA SDE approximation}

The purpose of this section is in establishing a framework for the stability analysis of Eq. (14) for the $m$ th moment stability concept presented in Definition 1. The $M n$-dimensional linear Ito SDE of Eq. (14) with an initial condition of $Y\left(t_{0}\right)=Y_{0}$ can be rewritten as

$d Y_{i}(t)=\mathbb{A}_{i j} Y_{j}(t) d t+\{\mathbb{G}\}_{i l k}\{Y(t)\}_{l} d \beta_{k}(t), \quad t \geq t_{0}$,

where $\mathbb{A}_{i j} Y_{j}(t)$ are the drift coefficients and $\{\mathbb{G}\}_{i l k}\{Y(t)\}_{l}$ are the diffusion coefficients.

The moment stability of Eq. (15) can be concluded if the moments of all orders are stable. However, there is an ambiguity in determining the stability of all moments since there are an infinite number of moments. For example, for a cer- 
tain values of the system parameters, a system can be stable for the first $p$ moments, where $p \geq 0$ represents the $p$ th moment stability, while the higher order moments, can be still unstable (see [8] for a more detailed example). Thus, we will only focus our attention on the study of the mean and mean-square stability of SDDEs. The other justification is that the mean-square stability condition implies almost sure stability condition for the special case of linear SDDEs as in Eq. $(15)[8,34,35]$. Thus, we can conclude that the meansquare stability can provide an acceptable measure for the stability analysis of Eq. (15). On the other hand, Eq. (15) is assumed to be excited by the Brownian motion increment process $d \beta(t)$ with the properties discussed in Sect. 2. Therefore, the moment equations, which will be obtained in this section, are deterministic which makes the stability analysis of Eq. (16) more tractable.

As discussed in [8], mean stability conditions are weak criteria since they do not yield any information about the sample stability. However, they can be considered as a necessary condition for the mean-square stability analysis. The mean-square stability analysis, on the other hand, can provide sufficient conditions for the stability of SDDEs. It is worth mentioning that determining the mean and mean-square stability conditions of Eq. (15) by using Definition 1 is also a challenging task. To analyze the stability of the system in the time domain, we employ the Ito differential rule to a scalar function.

Assume $\mathcal{F}(Y)$ is a scalar function which is twice differentiable with respect to the components of $Y(t)$. By applying the Ito differential rule to $\mathcal{F}(Y)$ along Eq. (15) and assuming that the correlation matrix $Q$ is a constant matrix, we can obtain

$d \mathcal{F}(Y)=\left(\mathcal{L}_{Y} \mathcal{F}\right) d t+\frac{\partial \mathcal{F}}{\partial Y_{i}}\left(\{\mathbb{G}\}_{i l k}\{Y(t)\}_{l}\right) d \beta_{k}(t)$,

where $\mathcal{L}$ is the generating differential operator of $Y$ defined as

$\mathcal{L}_{Y}=\left(\mathbb{A}_{i j} Y_{j}(t)\right) \frac{\partial}{\partial Y_{i}}+\frac{1}{2} \sigma_{i k} \sigma_{z k} \frac{\partial^{2}}{\left(\partial Y_{i} \partial Y_{z}\right)}$,

where $\sigma_{i k} \sigma_{z k}=\mathbb{G}_{i k} Q_{k r} \mathbb{G}_{z k}, r=1,2, \ldots, q$ and $\mathbb{G}_{i k}=$ $\{\mathbb{G}\}_{i l k}\{Y(t)\}_{l}$. Taking the expected value of Eq. (16) yields

$\frac{d E\{\mathcal{F}(Y)\}}{d t}=E\left\{\mathcal{L}_{Y} \mathcal{F}(Y)\right\}$.

Note that since obtaining the stability conditions of linear SDDEs either analytically or numerically using Definition 1 is a challenging task, the scalar function $\mathcal{F}$ and the operator $\mathcal{L}$ are employed to investigate the stability of the system.

Now as in [34], let us consider $\mathcal{F}$ to be

$\mathcal{F}(Y)=\mathbb{Y}_{1}^{s_{1}} \mathbb{Y}_{2}^{s_{2}} \cdots \mathbb{Y}_{(M n)}^{s_{(M n)}}$ where $s_{i}, i=1,2, \ldots,(M n)$ are nonnegative integers and $m=\sum_{i=1}^{M n} s_{i}$ is a positive integer. Keeping $m$ fixed and trying different compositions of $s_{i}$ powers, yields a set of deterministic equations for various $m$ th moments. Placing this set in a vector form, results in a new vector $\mathcal{F}(Y)$ of various $m$ th moment equations for Eq. (15) as

$\mathcal{F}(Y)=\left[\mathcal{F}_{1}, \mathcal{F}_{2}, \ldots, \mathcal{F}_{P}\right]$,

where $\mathcal{F}_{i}, i=1,2, \ldots, P, P=M n \frac{m(m+1)}{2}$ are components of the vector $\mathcal{F}$ and obtained by trying different compositions of $s_{i}$ powers in Eq. (19). Differentiating the elements of Eq. (20) using the Ito differential rule defined in Eq. (16), we can obtain a system of $P$ first order linear ordinary differential equations in a vector form for the various $m$ th moments which in general can be expressed as

$\frac{d E\{\mathcal{F}(Y)\}}{d t}=\mathcal{A} E\{\mathcal{F}(Y)\}$,

where $\mathcal{A}$ is a constant matrix of the elements of $\mathbb{A}_{i j}$, and $\mathbb{G}_{i l k}$. Then we can conclude that Eq. (15) is $m$ th moment asymptotically stable if the eigenvalues of $\mathcal{A}$ in Eq. (21) are in the left half plane. It should be noted that the $m$ th moment asymptotic stability of Eq. (15) can be also investigated using other stability methods such as the Lyapunov method or RouthHurwitz criteria.

Since in this paper we are interested in the mean and meansquare stability analysis of Eq. (15), the following two subsections devoted to stability analysis of the mean and meansquare in more detail.

\subsection{Mean stability condition}

To obtain the mean stability condition of Eq. (15), we set $m=1$ and $\mathcal{F}$ from Eq. (20) is defined as

$\mathcal{F}(Y)=\left[\mathbb{Y}_{1}, \mathbb{Y}_{2}, \ldots, \mathbb{Y}_{(M n)}\right]$.

Differentiating elements of Eq. (22) using the Ito differential rule expressed in Eq. (16) by considering the fact that $\frac{\partial^{2}}{\left(\partial \mathbb{Y}_{i} \partial \mathbb{Y}_{z}\right)}=0$ and the taking the expected value of the resulting equation, we can obtain

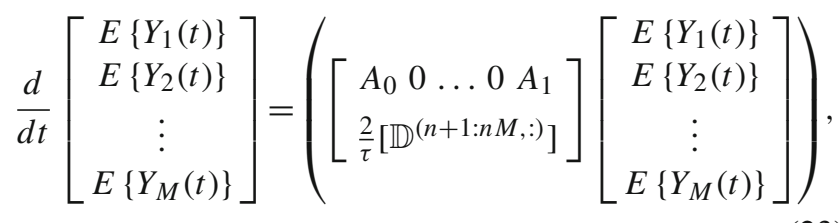

which can be rewritten as

$\frac{d E\{\mathcal{F}(Y)\}}{d t}=\mathcal{A} E\{\mathcal{F}(Y)\}$. 
where

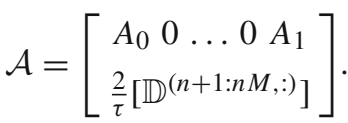

Thus the mean stability condition of the SDEs is guaranteed if the eigenvalues of $\mathcal{A}$ are in the left half plane.

As we discussed in this section, the mean stability is a weak criterion for the stability analysis of SDDEs. However, it can provide a necessary condition for the mean-square asymptotic stability. The following subsection discusses the mean-square asymptotic stability in more detail.

\subsection{Mean square stability}

The mean square stability condition can be obtained if we choose $m=2$ and the vector of mean-square moments $\mathcal{F}$ from Eq. (20) as

$$
\begin{aligned}
\mathcal{F}(Y)= & {\left[\mathbb{Y}_{1}^{2}, \mathbb{Y}_{1} \mathbb{Y}_{2}, \ldots \mathbb{Y}_{1} \mathbb{Y}_{(M n)}, \mathbb{Y}_{2}^{2}, \mathbb{Y}_{2} \mathbb{Y}_{3}, \ldots,\right.} \\
& \left.\mathbb{Y}_{2} \mathbb{Y}_{(M n)}, \ldots, \mathbb{Y}_{(M n)}^{2}\right]
\end{aligned}
$$

For applying Eq. (16) to the elements of Eq. (26), the following terms need to be obtained in advance:

$$
\begin{aligned}
& \frac{\partial \mathcal{F}(Y)}{\partial \mathbb{Y}_{i}}=\left[\begin{array}{c}
\mathcal{F}_{1}=\frac{\partial \mathbb{Y}_{1}^{2}}{\partial \mathbb{Y}_{i}} \\
\mathcal{F}_{2}=\frac{\partial \mathbb{Y}_{1} \mathbb{Y}_{2}}{\partial \mathbb{Y}_{i}} \\
\vdots \\
\mathcal{F}_{P}=\frac{\partial \mathbb{Y}_{(M n)}^{2}}{\partial \mathbb{Y}_{i}}
\end{array}\right] \\
& =\left[\begin{array}{cccccc}
2 \mathbb{Y}_{1} & 0 & 0 & 0 & \ldots & 0 \\
\mathbb{Y}_{2} & \mathbb{Y}_{1} & 0 & 0 & \ldots & 0 \\
\vdots & & & \ddots & & \vdots \\
0 & 0 & 0 & 0 & \ldots & 2 \mathbb{Y}_{(M n)}
\end{array}\right]_{P \times(M n)}
\end{aligned}
$$

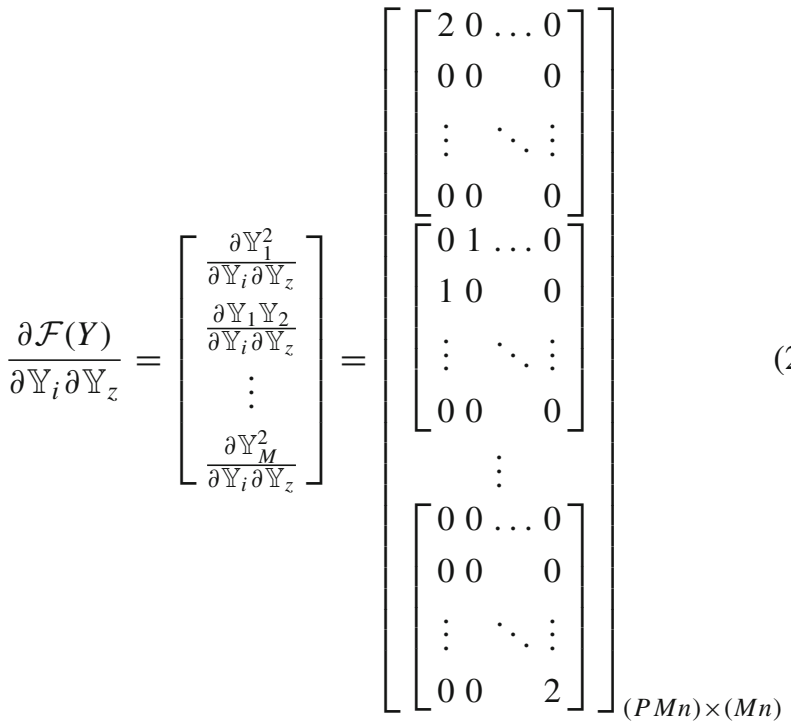

$$
\mathcal{F}(Y)=\mathbb{Y}_{1}^{s_{1}} \mathbb{Y}_{2}^{s_{2}} \ldots \mathbb{Y}_{6}^{s_{6}}
$$

where $s_{1}+s_{2}+\cdots+s_{6}=2$. The moment stability of the system can be studied by means of the eigenvalues of the second moment differential equation.

A convergence study shows that the sufficient number of collocation points for the mean square stability boundaries to converge with visually indistinguishable results is $N=5$ 
Fig. 1 The convergence of the mean square stability boundaries for the stochastic DDE of Eq. (30) with $\tau=1$ (s) shows 5 Chebyshev collocation points is sufficient for visually indistinguishable results

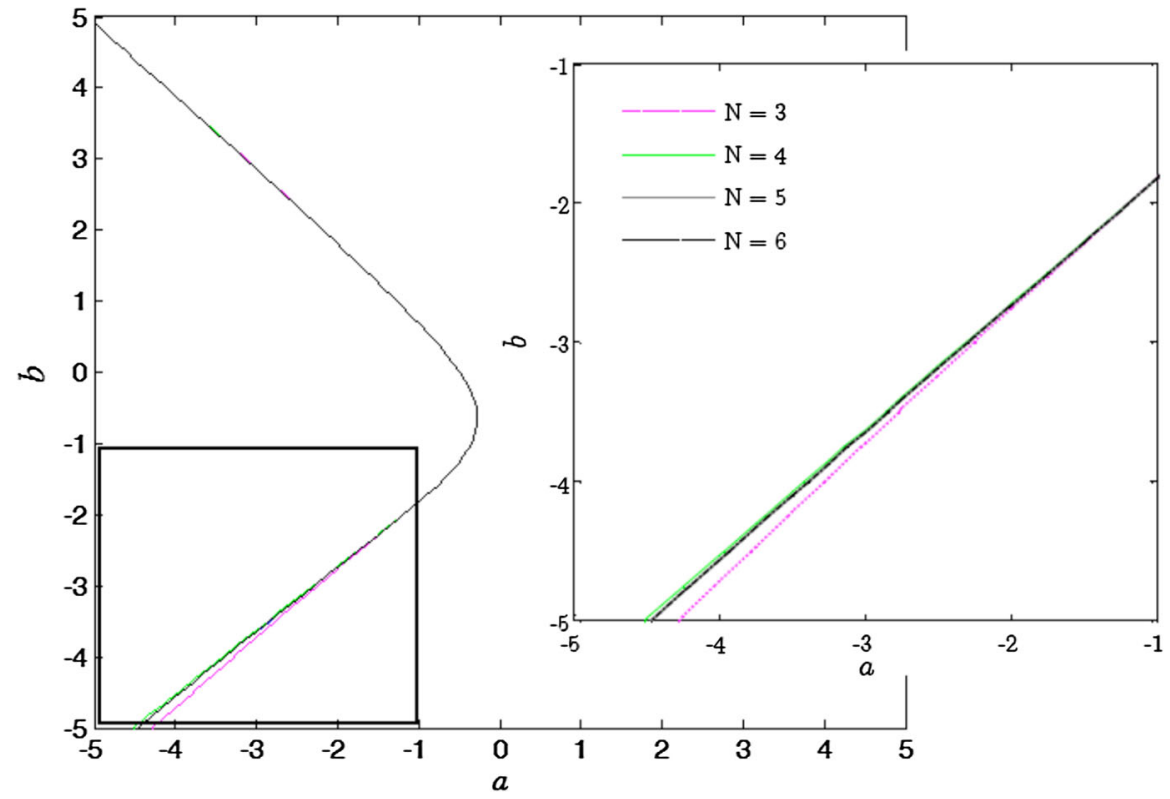

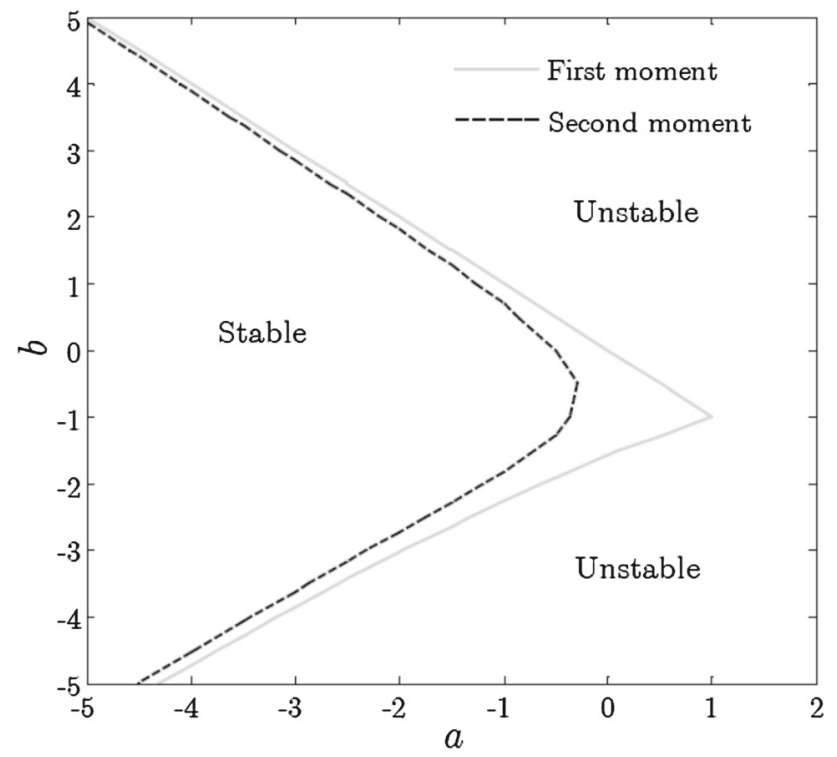

Fig. 2 The mean and mean square stability boundaries for the stochastic DDE system of Eq. (30) with $\tau=1$ (s) obtained from both CSCTA $(N=5$ points used) and the stochastic semi-discretization method ( $N=10$ points used), visually indistinguishable from CSCTA result

(Fig. 1). This is in fact owing to the exponential convergence characteristics of CSCTA. The mean and mean square stability boundaries for the SDDE of Eq. (30) obtained from CSCTA and the stochastic semi-discretization method [1] with 10 points used for equivalent accuracy are depicted in Fig. 2.

The above results are now compared with the stability charts obtained using a Lyapunov-based approach in [2]. This comparison is depicted in Fig. 3. As is expected, the

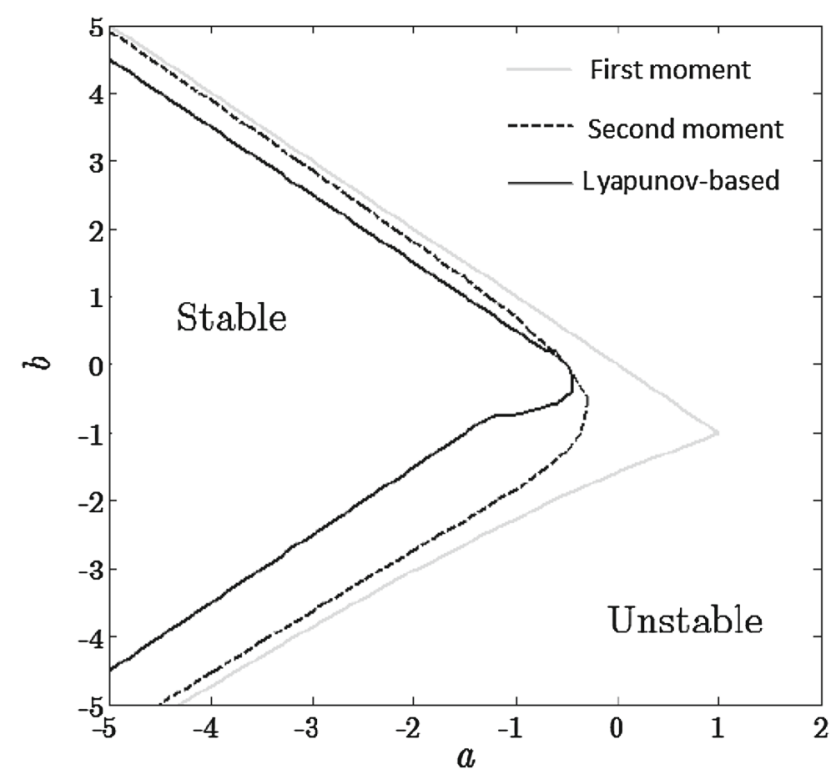

Fig. 3 The mean and mean square stability boundaries for stochastic DDE of Eq. (30) compared with the Lyapunov-based stability result of [2]

stability boundaries from the current numerical approach are less conservative than those from the Lyapunov-based approach. This observation is in agreement with the comparison made in [2] between the Lyapunov-based stability chart for the first order system of Eq. (30) with the numerical stability chart for this system obtained using the stochastic semi-discretization approach with $n=10$ discretization points, which yields identical stability results with the proposed CSCTA-based approach for this particular example. It should be noted that more discretization points are required 
for convergence using stochastic semi-discretization than are required using CSCTA.

\subsection{Second order linear stochastic time-delayed system}

In this section, we consider the second order linear timedelayed system in [1] with both additive and multiplicative stochastic excitations expressed in the physical form as

$$
\begin{aligned}
\dot{x}(t)= & {\left[\begin{array}{c}
x_{2} \\
-a_{1} x_{2}(t)-a_{2} x_{1}(t)-k_{p} x_{1}(t-\tau)-k_{d} x_{2}(t-\tau)
\end{array}\right] } \\
& +\left[\begin{array}{ccc}
0 & 0 & 0 \\
x_{1}(t) & x_{2}(t) & 1
\end{array}\right] w(t)
\end{aligned}
$$

where $a_{1}$ and $a_{2}$ are constant parameters, $k_{p}$ and $k_{d}$ can be considered as delay feedback control gains, and $w(t)$ is the white noise process which approximates the wide-band process $\zeta(t)$ with $w(t) \sim(0, Q(t))$ and $Q(t)$ is a $3 \times 3$ covariance matrix. Eq. (32) can be converted to the Ito differential form of Eq. (4) by adding the Wong-Zakai correction term as

$$
\begin{aligned}
d x(t)= & {\left[\begin{array}{c}
x_{2} \\
-a_{1} x_{2}(t)-a_{2} x_{1}(t)+\pi\left(Q_{22} x_{2}(t)+Q_{12} x_{1}(t)\right. \\
\left.+Q_{23}\right)-k_{p} x_{1}(t-\tau)-k_{d} x_{2}(t-\tau)
\end{array}\right] d t } \\
& +\left[\begin{array}{ccc}
0 & 0 & 0 \\
x_{1}(t) & x_{2}(t) & 1
\end{array}\right] d \beta(t) .
\end{aligned}
$$

In order to be able to compare the stability in the $k_{p}-k_{d}$ parameter space with that from the stochastic semi-discretization method in [1], we consider the case where $\tau=0.16$, $a_{1}=0.4, a_{2}=1, Q_{22}=0.5 \sqrt{2 \pi}, Q_{11}=\sqrt{2 \pi}$ and $Q_{33}=Q_{12}=Q_{13}=Q_{23}=0$.

The procedure described in Sect. 4 is applied for numerical stability analysis of the time-delayed system of Eq. (33). The convergence of the stability boundaries are visually depicted in Fig. 4. For a numerical convergence study of the stability boundaries, one arbitrary point inside the boundary and one outside is picked and the percentage relative difference of $\lambda_{\max }(\mathcal{A})$ as $N$ increases is calculated, where $\lambda_{\max }(\mathcal{A})$ is the maximum eigen-values for matrix $\mathcal{A}$. For each of those points the number of collocation points are increased until it converges to within the tolerance of \pm 0.01 (Table 1 ). The numerical convergence study confirms the conclusion from Fig. 4 that the stability boundaries are converged by using only 7 collocation points. The resulting stability boundary is depicted in Fig. 5. Also in this figure, the stability boundary obtained from the current approach is compared with the one obtained from the stochastic semi-discretization method. As it can be seen from Fig. 5, the second moment stability boundaries for the system of Eq. (32) obtained from the current approach almost match the stability boundaries obtained from semi-discretization approach described in [1] (Table 2).

It is worthy of noting that when applying the semidiscretization method for the system of Eq. (33) we noticed that the number of discretization points required for the stability boundaries to converge to within a tolerance of $\pm 0.01 \%$ is 25. Therefore, the CSCTA approach has better convergence properties for this example as it converges to the same accuracy with 7 collocation points (Fig. 4). Also, as was discussed for the first order example, the stability results based on CSCTA approach converge with 5 collocation points (Fig.
Fig. 4 Mean square stability boundaries versus the number of collocation points used in CSCTA, $N=7$ is sufficient for visually indistinguishable results

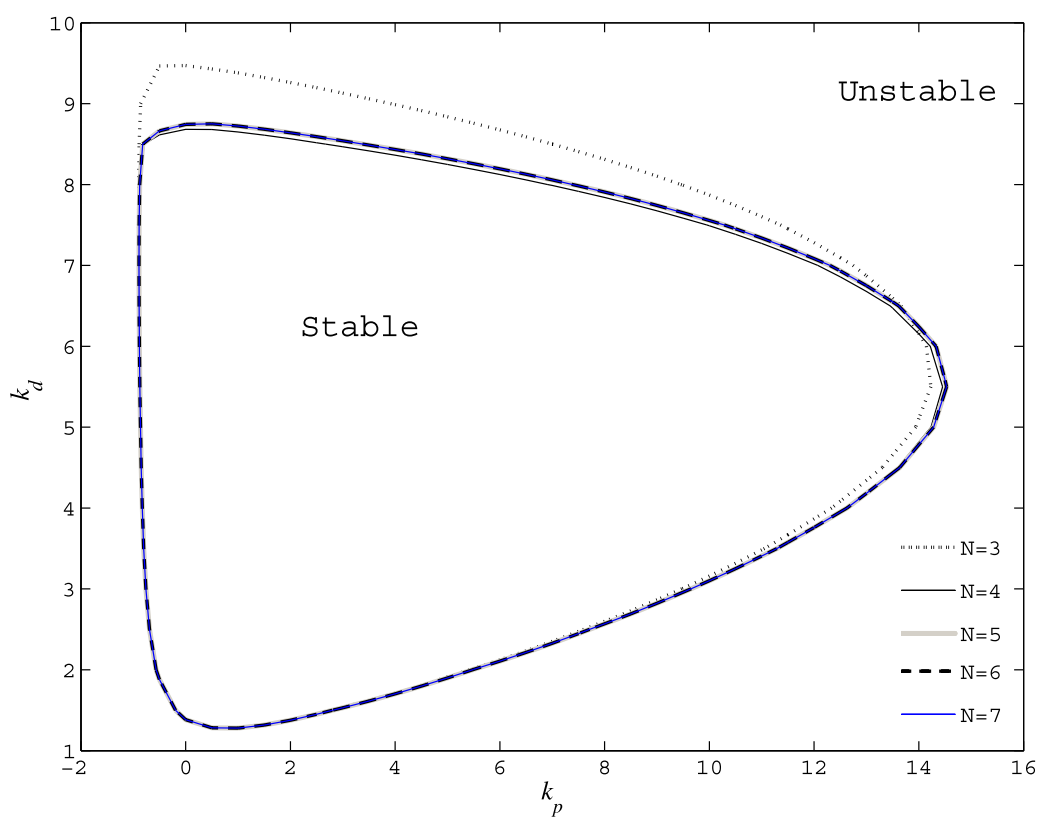


Table 1 Numerical convergence study of two arbitrary points on the stability boundaries of the system of Eq. (33) using CSCTA method

\begin{tabular}{llclll}
\hline$N$ & \multicolumn{2}{l}{$k_{p}=14.5, k_{d}=5.5$} & & \multicolumn{2}{l}{$k_{p}=15.0, k_{d}=5.5$} \\
\cline { 2 - 3 } \cline { 5 - 6 } & $\lambda_{\max }(\mathcal{A})$ & Relative difference $(\%)$ & & $\lambda_{\max }(\mathcal{A})$ & Relative difference $(\%)$ \\
\hline 2 & 0.05597092 & & 0.15790497 & \\
3 & 0.01005142 & -82.042 & 0.11921425 & -24.503 \\
4 & -0.00680159 & -167.668 & 0.10269691 & -13.855 \\
5 & -0.00672475 & -1.130 & 0.10274626 & 0.048 \\
6 & -0.00667274 & -0.773 & 0.10279688 & 0.049 \\
7 & -0.00666808 & -0.070 & 0.10280144 & 0.004 \\
8 & -0.00666518 & -0.043 & 0.10280422 & 0.003
\end{tabular}

Fig. 5 The mean and mean square stability boundaries of the SDDE of Eq. (33) with $\tau=0.16, a_{1}=0.4, a_{2}=1$, $Q_{22}=0.5, Q_{11}=1$ and $Q_{33}=Q_{12}=Q_{13}=Q_{23}=0$ obtained from both CSCTA $(N=7)$ and the stochastic semi-discretization method $(N=25)$ see Tables 1 and 2

Table 2 Numerical convergence study of two arbitrary points on the stability boundaries of the system of Eq. (33) using semi-discretization method

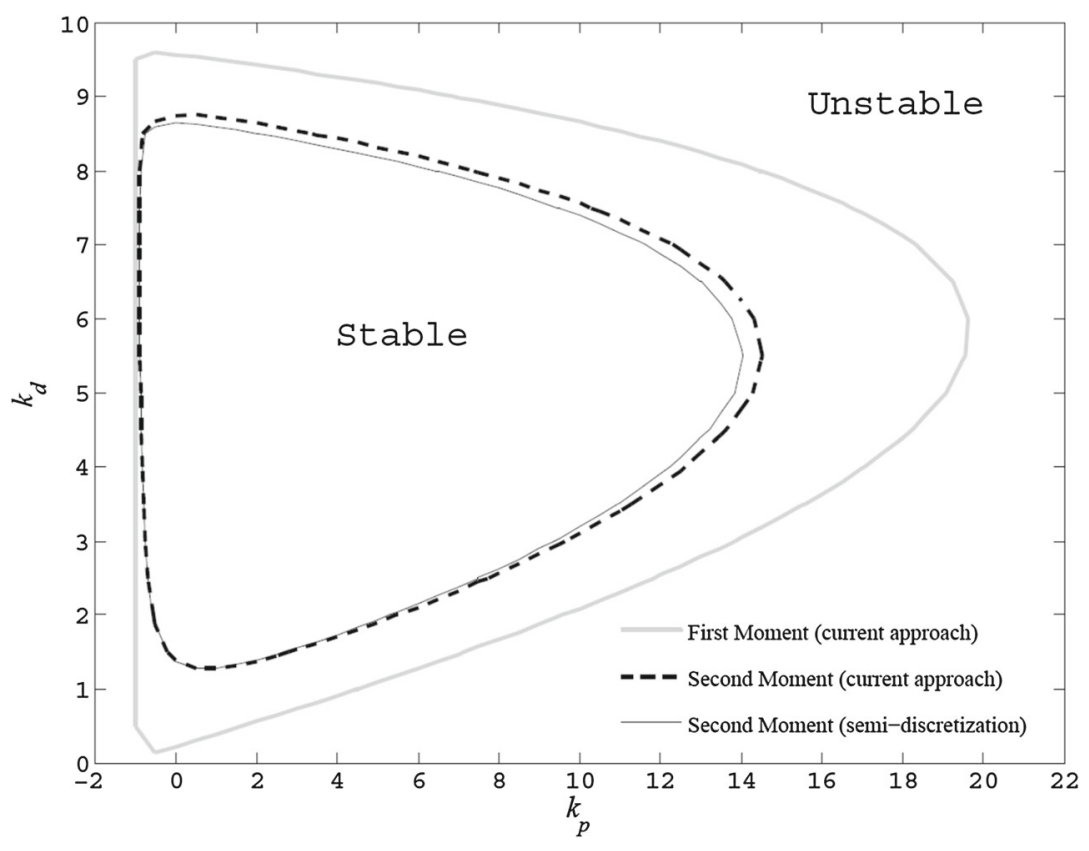

\begin{tabular}{|c|c|c|c|c|}
\hline \multirow[t]{2}{*}{$N$} & \multicolumn{2}{|c|}{$k_{p}=14.0, k_{d}=5.5$} & \multicolumn{2}{|c|}{$k_{p}=14.5, k_{d}=5.5$} \\
\hline & $\lambda_{\max }(\mathcal{A})$ & Relative difference $(\%)$ & $\lambda_{\max }(\mathcal{A})$ & Relative difference $(\%)$ \\
\hline 5 & 1.01939731 & & 1.02284033 & \\
\hline 10 & 1.00425748 & -1.485 & 1.00602919 & -1.644 \\
\hline 15 & 1.00152256 & -0.272 & 1.00271846 & -0.329 \\
\hline 20 & 1.00063108 & -0.089 & 1.00153411 & -0.118 \\
\hline 25 & 1.0002549 & -0.038 & 1.00098043 & -0.055 \\
\hline 30 & 1.00007184 & -0.018 & 1.00067822 & -0.030 \\
\hline 35 & 0.99997477 & -0.010 & 1.00049564 & -0.018 \\
\hline 36 & 0.99996135 & -0.001 & 1.00046793 & -0.003 \\
\hline
\end{tabular}

1), while at least 10 discretization points are required for an equivalent accuracy using semi-discretization approach. This signifies the advantage of exponential convergence of the current approach over the stochastic semi-discretization method.

\section{Conclusion}

A numerical approach was developed for stability analysis of linear SDDEs based on the CSCTA technique. Using CSCTA, the infinite-dimensional linear SDDE was approxi- 
mated by a set of linear SDEs. Then, the $m$ th moment stability concept was utilized and a set of first order linear differential equations for both the mean and mean-square stability conditions were obtained. Two examples were studied and the stability charts obtained from the proposed approach were compared with those produced by both Lyapunov-based method in [2] and the stochastic semi-discretization method in [1]. As expected, the numerical stability regions using the proposed approach were found to be less conservative compared with those obtained from the Lyapunov-based approach, while they agreed with results obtained using the stochastic semi-discretization method. In the case of the second order SDDE, the second moment stability region obtained using CSCTA was found to give almost identical results compared to the semi-discretization approach described in [1]. The advantage of this proposed method over the stochastic semi-discretization method is the exponential convergence characteristic of the spectral approximation technique used in this paper. This fact is clearly shown in both examples in which less Chebyshev collocation points are required for convergence compared to the number of points used for convergence of the stochastic semi-discretization approach.

Acknowledgments Financial support from the National Science Foundation under Grant No. CMMI-0900289 is gratefully acknowledged.

\section{References}

1. Elbeyli O, Sun J, Ünal G (2005) A semi-discretization method for delayed stochastic systems. Commun Nonlinear Sci Numer Simul 10(1):85-94

2. Samiei E, Torkamani S, Butcher EA (2013) On Lyapunov stability of scalar stochastic time-delayed systems. Int J Dyn Control 1(1):64-80

3. Sobczyk K (2001) Stochastic differential equations: with applications to physics and engineering, vol 40. Springer, Berlin

4. Jokipii JR, Parker EN (1969) Stochastic aspects of magnetic lines of force with application to cosmic-ray propagation. Astrophys J 155:777-798

5. Primak S, Kontorovitch V, Lyandres V (2005) Stochastic methods and their applications to communications: stochastic differential equations approach. Wiley, New Jersey

6. Li M, Zhou X, Rouphail N (2011) Quantifying benefits of traffic information provision under stochastic demand and capacity conditions: A multi-day traffic equilibrium approach, In: 14th International IEEE Conference on Intelligent Transportation Systems (ITSC), pp 2118-2123

7. Meditch JS (1969) Stochastic optimal linear estimation and control. McGraw-Hill, New York

8. Ibrahim RA (2007) Parametric random vibration. Dover Publication Inc., New York

9. Jazwinski AH (2007) Stochastic processes and filtering theory. Dover Publications, Mineola

10. Masoller C (2002) Noise-induced resonance in delayed feedback systems. Phys Rev Lett 88(3):034102

11. Mao X (1992) Robustness of stability of nonlinear systems with stochastic delay perturbations. Syst Control Lett 19(5):391-400
12. Fofana M (2002) Asymptotic stability of a stochastic delay equation. Probab. Eng. Mech. 17(4):385-392

13. Mao X, Koroleva N, Rodkina A (1998) Robust stability of uncertain stochastic differential delay equations. Syst Control Lett 35(5):325-336

14. Mackey MC, Nechaeva IG (1995) Solution moment stability in stochastic differential delay equations. Phys Rev E 52(4):3366

15. Mohammed S (1986) Stability of linear delay equations under a small noise. Proc Edinb Math Soc 2 29(02):233-254

16. Chen W-H, Guan Z-H, Lu X (2005) Delay-dependent exponential stability of uncertain stochastic systems with multiple delays: an Lmi approach. Syst Control Lett 54(6):547-555

17. He Y, Wu M, She J-H, Liu G-P (2004) Parameter-dependent lyapunov functional for stability of time-delay systems with polytopictype uncertainties. IEEE Trans Autom Control 49(5):828832

18. Mackey MC, Nechaeva IG (1994) Noise and stability in differential delay equations. J Dyn Differ Equ 6(3):395-426

19. Nechaeva I, Khusainov D (1990) Exponential estimates of solutions of linear stochastic differential functional equations. Ukr Math J 42:1189-1193

20. Garay BM (2005) A brief survey on the numerical dynamics for functional differential equations. Int J Bifurcat Chaos 15(03):729_ 742

21. Győri I, Hartung F, Turi J (1998) Preservation of stability in delay equations under delay perturbations. J Math Anal Appl 220(1):290-312

22. Insperger T, Stépán G (2002) Semi-discretization method for delayed systems. Int J Numer Methods Eng 55(5):503-518

23. Trefethen LN (2000) Spectral methods in MATLAB, vol 10. Siam, Philadelphia

24. Butcher EA, Bobrenkov OA (2011) On the Chebyshev spectral continuous time approximation for constant and periodic delay differential equations. Commun Nonlinear Sci Numer Simul 16(3):1541-1554

25. Bobrenkov OA, Nazari M, Butcher EA (2012) Response and stability analysis of periodic delayed systems with discontinuous distributed delay. J Comput Nonlinear Dyn 7(3):031010

26. Torkamani S, Butcher EA (2013) Delay, state, and parameter estimation in chaotic and hyperchaotic delayed systems with uncertainty and time-varying delay. Int J Dyn Control 1(2):1-29

27. Torkamani S, Butcher EA (2013) Stochastic parameter estimation in nonlinear time-delayed vibratory systems with distributed delay. J Sound Vib 332(14):3404

28. Torkamani S, Butcher EA (2013) Optimal parameter and state estimation in stochastic time-varying systems with time delay. Commun Nonlinear Sci Numer Simul 18(8):2188

29. Breda D, Maset S, Vermiglio R (2005) Pseudospectral differencing methods for characteristic roots of delay differential equations. SIAM J Sci Comput 27(2):482-495

30. Breda D, Maset S, Vermiglio R (2012) Approximation of eigenvalues of evolution operators for linear retarded functional differential equations. SIAM J Numer Anal 50(3):1456-1483

31. Stratonovich R (1966) A new form of representing stochastic integrals and equations. SIAM J Control 4:362-371

32. Taniguchi T, Liu K, Truman A (2002) Existence, uniqueness, and asymptotic behavior of mild solutions to stochastic functional differential equations in hilbert spaces. J Differ Equ 181(1):72-91

33. Xu D, Yang Z, Huang Y (2008) Existence-uniqueness and continuation theorems for stochastic functional differential equations. $\mathrm{J}$ Differ Equ 245(6):1681-1703

34. Lin Y-K, Cai G-Q (1995) Probabilistic structural dynamics: advanced theory and applications. McGraw-Hill, New York

35. Mitchell RR, Kozin F (1974) Sample stability of second order linear differential equations with wide band noise coefficients. SIAM J Appl Math 27(4):571-605 
36. Bátkai A, Piazzera S (2005) Semigroups for delay equations. AK Peters, Wellesley

37. Michiels W, Niculescu S-I (2007) Stability and stabilization of time-delay systems: an eigenvalue-based approach (advances in design and control). Soc Ind Appl Math

38. Hale JK, Lunel SMV (1993) Introduction to functional differential equations, vol 99. Springer, Berlin

39. Diekmann O, van Gils SA, Lunel SV, Walther H-O (1995) Delay equations: functional-, complex-, and nonlinear analysis. SpringerVerlag, New York
40. Van Neerven J, Weis L (2005) Stochastic integration of functions with values in a banach space. Stud Math 166(2):131-170

41. Van Neerven J, Riedle M (2007) A semigroup approach to stochastic delay equations in spaces of continuous functions. Semigroup Forum 74:227-239

42. Cox S, Górajski M (2011) Vector-valued stochastic delay equationsa semigroup approach. Semigroup Forum 82:389-411

43. Fox L, Parker IB (1968) Chebyshev polynomials in numerical analysis, vol 29. Oxford University Press, London 